\title{
Muography with nuclear emulsions - Stromboli and other projects
}

Valeri Tioukov $^{1,2,{ }^{\star}}$, Giovanni De Lellis ${ }^{1,2}$, Paolo Strolin ${ }^{1,2}$, Lucia Consiglio ${ }^{1,2}$, Andrey Sheshukov ${ }^{1,2}$, Massimo Orazi ${ }^{3}$, Rosario Peluso ${ }^{4,5}$, Cristiano Bozza ${ }^{4,5}$, Chiara De Sio ${ }^{4,5}$, Simona Maria Stellacci ${ }^{4,5}$, Chiara Sirignano ${ }^{6,7}$, Nicola D’Ambrosio ${ }^{8}$, Seigo Miyamoto ${ }^{9}$, Ryuichi Nishiyama ${ }^{9}$, Hiroyuki K.M. Tanaka ${ }^{9}$

\author{
${ }^{1}$ Università di Napoli Federico II, Dipartimento di Fisica, Naples, Italy \\ ${ }^{2}$ Istituto Nazionale di Fisica Nucleare (INFN), Sezione di Napoli, Naples, Italy \\ ${ }^{3}$ Istituto Nazionale di Geofisica e Vulcanologia (INGV), Sezione di Napoli - Osservatorio Vesuviano, Naples, Italy \\ ${ }^{4}$ Università di Salerno, Dipartimento di Fisica “E. R. Caianiello", Salerno, Italy \\ 5 Istituto Nazionale di Fisica Nucleare (INFN), Gruppo Collegato di Salerno, Salerno, Italy \\ ${ }^{6}$ Università di Padova, Dipartimento di Fisica e Astronomia "Galileo Galilei”, Padova, Italy \\ ${ }^{7}$ Istituto Nazionale di Fisica Nucleare (INFN), Sezione di Padova, Padova, Italy \\ ${ }^{8}$ Istituto Nazionale di Fisica Nucleare (INFN), Laboratori Nazionali del Gran Sasso, Assergi (L'Aquila), Italy \\ ${ }^{9}$ Earthquake Research Institute, The University of Tokyo, Tokyo, Japan
}

\author{
Article history \\ Received September 18, 2016; accepted October 5, 2016. \\ Subject classification: \\ Muon radiography, Nuclear emulsion, Stromboli, Volcano, Tracking detector.
}

\section{ABSTRACT}

The muon radiography is a novel imaging technique to probe the volcanoes interior, using the capability of high energy cosmic ray muons to penetrate large thicknesses of rock. In this way it is possible to derive a $2 \mathrm{D}$ density map along the muon trajectory of volcanic edifices and deduce information on the variations in the rock density distribution, like those expected from dense lava conduits, or low density magma supply paths. This method is applicable also to study geological objects as glaciers, faults, oil underground reservoirs, engineering constructions, where a density contrast is present. Nuclear emulsions are well suited to be employed in this context for their excellent angular resolution; they are compact and robust detectors, able to work in harsh environments without need of power supply. On the other side, a long exposure time is required for a reasonable detector surface $\left(\sim 10 \mathrm{~m}^{2}\right)$ in order to collect a sufficient statistics of muons, and a quasi-real time analysis of the emulsion data is rather difficult due to the scanning time needed by the optical microscopes. Such drawback is on the way to be overcome thanks to a recent RひD program on ultra-fast scanning systems. Muon radiography technique, even if limited to the summit part of the volcano edifice, represents an important tool of investigation, at higher spatial resolution, complementary to the conventional geophysics techniques. The first successful result in this field was obtained by a Japanese group that observed in 2007 the conduit structure of Mt. Asama. Since 2010, other interesting volcanoes have been probed with the same method: Stromboli in 2011, Mt. Teide in 2012 and La Palma in 2014. Here we discuss the muon imaging
\end{abstract}

technique reporting the nuclear emulsion detector design exposed at Stromboli and results of the data analysis.

\section{The muon radiography technique}

The internal structure of active volcanoes, is one of the most important geophysical questions. Currently, the shallow Earth crust is explored in great detail with echo-sounding, electromagnetic or gravimetric techniques, but it cannot be well resolved because of its strong structural heterogeneity and the potential difficulty to be accessed. Actually such indirect methods suffer from inherent ambiguity, require spatially dense measurements in active areas and may not provide sufficient spatial resolution in the uppermost part of the conduit system. The muon radiography performed with cosmic-ray muons represents a complementary method able to provide a direct snapshot of the density profile in a volcano. In someway it is similar to the standard medical radiography: it allows to view inside volcanoes from a certain distance, thus reducing the risk for the operator, using instead of X-rays, atmospheric muon particles from cosmic rays. Cosmic muons are the products of pion and kaon decays originated from the hadronic interaction of the primary cosmic rays (mainly protons and alpha particles) with the atmos- 


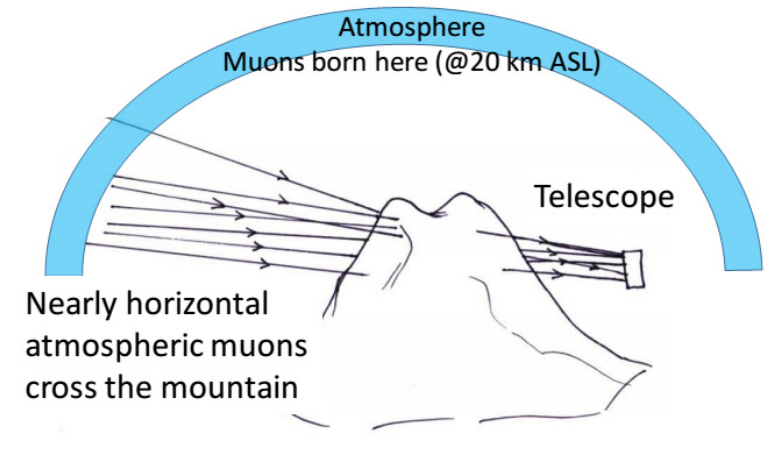

Figure 1. The principle of muography.

pheric nuclei. Muons are the charged particles with the highest penetration capability in the matter. Their mass (about 200 times the electron mass) and their long lifetime allow them to propagate through the layers of the atmosphere toward the Earth's ground from any direction. Cosmic rays reach very high energies, which are partially inherited by the muons. The muon average energy at the sea level is around $4 \mathrm{GeV}$ near the zenith [Gaisser 1990], but there are very large tails, up to several TeV. The muon flux intensity and energy distribution depend on the zenith angle. The nearly horizontal muons, are more interesting for the radiography purpose because their higher energy, allows them penetrate larger rock thicknesses.

A muon detector is usually positioned on a slope of the volcano as schematically shown in Figure 1, pointing toward a prominent rock region of interest in such a way it's possible to derive results for the rock portion located above the detector. Typically the measured fluxes are relatively low and strongly dependent on the amount of the thickness crossed. For instance, a muon telescope with a surface of the order of $1 \mathrm{~m}^{2}$ with 1 steradian aperture angle can record about $10^{3}$ muons per day crossing a rock thickness equivalent to $2 \mathrm{~km}$ of water. The energy spectrum of the atmospheric muons is well known; thus assuming also the precise knowledge of the mountain shape and a well understood muon detector it is possible to infer a map of the rock density distribution, by using the informations on the attenuation and the absorption of the muon flux. Since denser materials absorb more muons (like dense materials such as bones absorb more Xrays), this provides a basis for producing shadow images of the volcano interior. An important advantage of the technique is the spatial resolution that can be achieved: whereas current indirect methods can provide information with a spatial resolution of some hundreds of meters, muon radiography may provide mapping of internal structures to a resolution of some tens of meters. The resolution in solving the density contrast depends on the thickness of the rock traversed by the muons: the thicker it is, the fainter the muon flux is and the longer it takes to collect enough muons for a picture. The time needed can thus range from some weeks to several months. Another interesting feature of this technique, is the possibility to perform a tomographic measurement by placing two or more cosmic ray telescopes around the object of interest.

\section{Nuclear emulsions for muon recording}

Mainly there are two kind of detectors that can be used for muon radiography purpose: real time detectors (like plastic scintillators or wire chambers) and nuclear emulsion films used in our observations. Emulsion detectors, have an incomparable position and intrinsic angular resolution (less than $1 \mu$ and a $1 \mathrm{mrad}$, respectively), high data storage capabilities, mechanical robustness and compactness. They are not real time detectors and differently from the electronic ones do not require power supply and electronic front-end readout systems. Moreover, their quite simple implementation in harsh environments like the ones encountered in active zones of a volcano and their easy portability, makes them ideal for geological applications. The very recent $\mathrm{R} \& \mathrm{D}$ program on new generation faster scanning systems currently in use for the OPERA experiment on neutrino oscillation search [Acquafredda et al. 2009; Agafonova et al. 2010, 2013, 2014] have recently encouraged the employment of nuclear emulsions as tracking detector in this field allowing to exploit widely their potential.

The nuclear emulsion films used in our detector are made of two layers $45 \mu \mathrm{m}$ thick of $\mathrm{AgBr}$ crystals suspended in an organic gelatine poured on a plastic base of $200 \mu \mathrm{m}$. When a charged particle crosses the sensitive layer, some micro-crystals on the particle trajectory record its path as sequence of dark aligned grains $(0.3-1 \mu \mathrm{m}$ diameter) as shown in Figure 2 which can be analyzed after a chemical development of the films, by an optical microscope. The grain density in the emulsion is about $35 / 100 \mu \mathrm{m}$ optimized for a min-

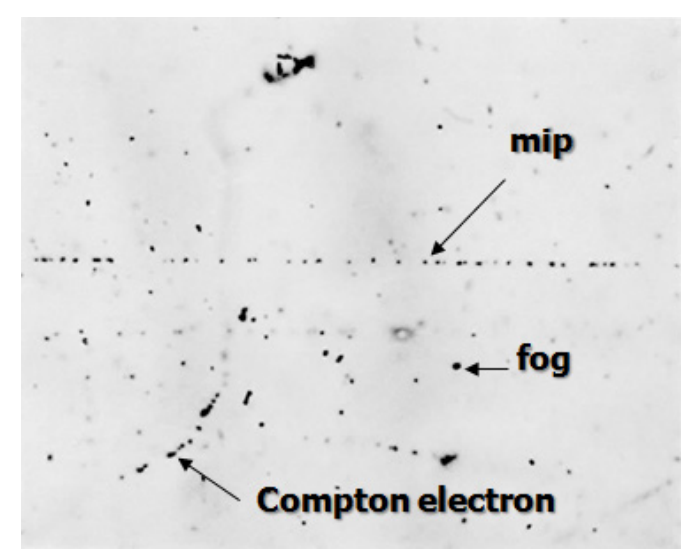

Figure 2. Cross sectional view of a nuclear emulsion layer. 


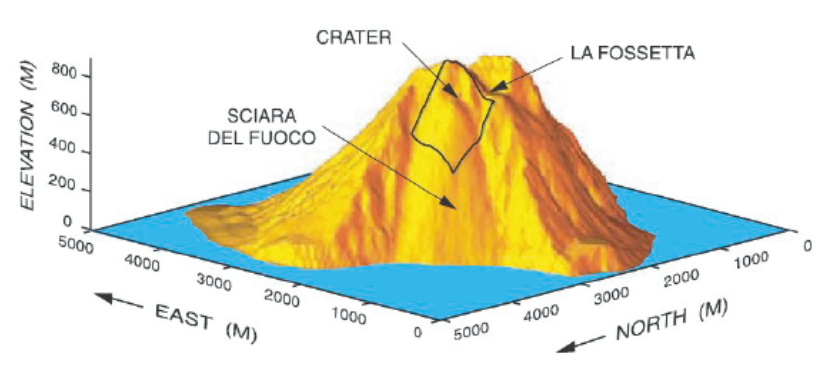

Figure 3. A south-east view of Stromboli. The Sciara del Fuoco region and the active craters are indicated.

imum ionizing particle. As final response, the detector will provide a precise measurement of the incident angle of the muons. A detailed description of the nuclear emulsion detector is given in Nakamura et al. [2006]. The first experiments of muon radiography of volcanoes with nuclear emulsion technique have been successfully conducted on Mt. Asama [Tanaka et al. 2007a] and Showa-Shinzan [Tanaka et al. 2007b] in Japan. After that, new emulsion projects involving Italian laboratories have been carried on: Stromboli volcano in 2011, Teide mountain radiography (2012) and La Palma exposure (2014) in Tenerife.

\section{The Stromboli survey}

Stromboli is a composite strato-volcano that steeply reaching $\sim 900 \mathrm{~m}$ above sea level, allows to image a large portion of the volcano edifice by muon radiography. The Stromboli activity is marked out by a shallow seismicity occurring at about $200 \mathrm{~m}$ depth below the cone [Chouet et al. 2007] associated with eruptions as well as the continuous volcanic tremor and intermittent explosions of gas jets and volcanic materials that are concentrated at depths shallower than $200 \mathrm{~m}$ beneath the summit crater [Auger et al. 2006]. One of the most interesting regions is the so-called Sciara del Fuoco since, according to some theoretical models of the volcanic activity, the main explosion mechanism would be due to some cracks opening from the main conduit of this region [Macedonio 2009].

An advanced geophysical system is installed on the volcano slopes, for monitoring several parameters (seismic, infrasounds, thermal infrared, ground deformation, chemical composition of fumaroles), but the precise internal structure of the region below craters is not completely understood yet. The muon radiography could be an independent tool for investigating the inner structure of the cone and revealing the location and extent of the conduits that feed the continuous explosions. The region where the structures are expected is indicated by a black line in Figure 3. Possible cracks or channels transverse dimensions is expected as 10 me- ters or less. This resolution could be reached by muon radiography in a favorable conditions.

\subsection{The muon telescope}

An emulsion telescope with an area of $0.96 \mathrm{~m}^{2}$ was installed in the end of 2011 at $640 \mathrm{~m}$ of altitude pointing toward the Sciara region. The apparatus consists of 8 modules (Figure 4) each one equipped with 10 emulsion cells as shown in Figure 5.

Each of them is made of 2 doublets of emulsion films individually vacuum-packed in light shield envelopes, coated on both sides of a central metal plate of $26 \mathrm{~cm} \times 80 \mathrm{~cm}$ size and $5 \mathrm{~mm}$ thick (Figure 6). The emulsion doublets are coated to the central plate by 2 layers of elastic rubber while additional inox steel plates integrate the module structure, in such way to guarantee flatness and at the same time shield the soft component of cosmic rays. The total amount of emulsion films per module is 40 . The total weight of one module is $26 \mathrm{~kg}$ while the overall weight of 8 modules including also the support frame is about $250 \mathrm{~kg}$. In order to avoid possible rotation effects of any emulsion film a rigid support frame was realized. The detector position and orientation was obtained by recording GPS data of two outer limits of the structure. The detector exposure went on for 5 months (from the end of October to the end of March); during this period, a continuous temperature

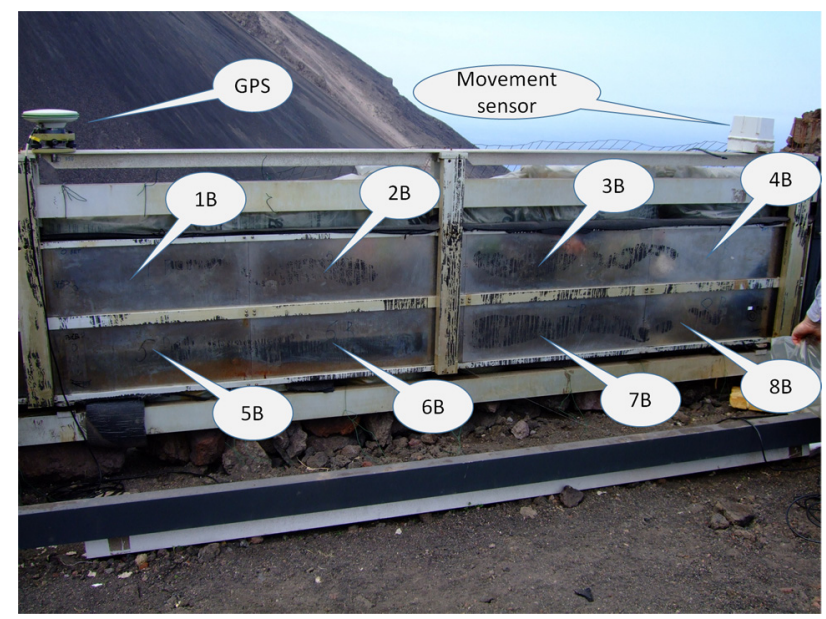

Figure 4. Picture of the detector structure.
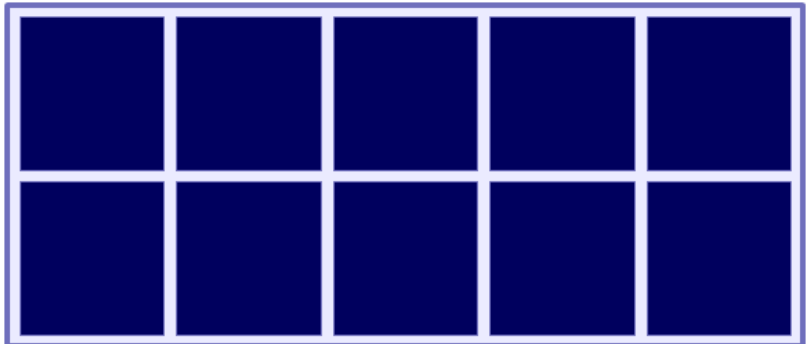

Figure 5. Front view of a single detector module. 


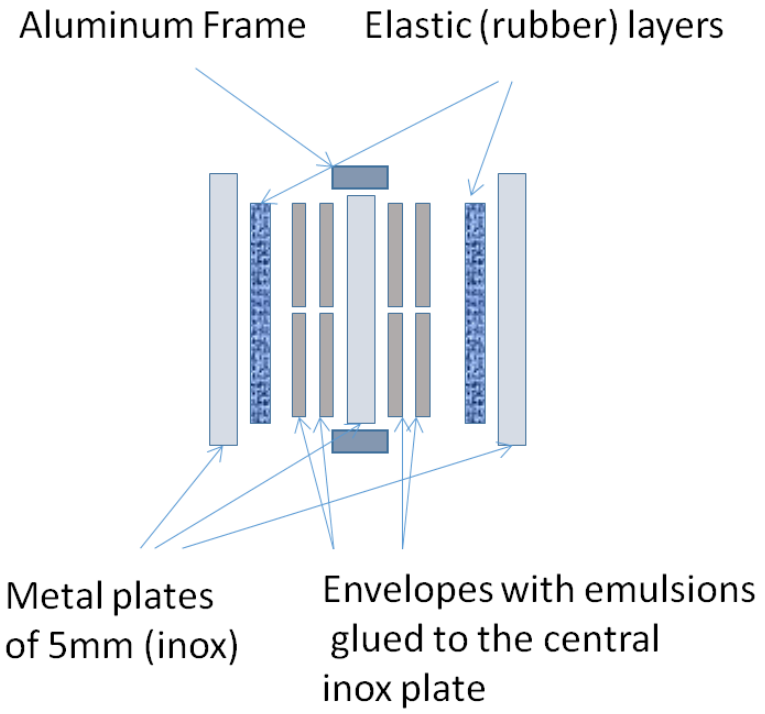

Figure 6. Side view of a single module.

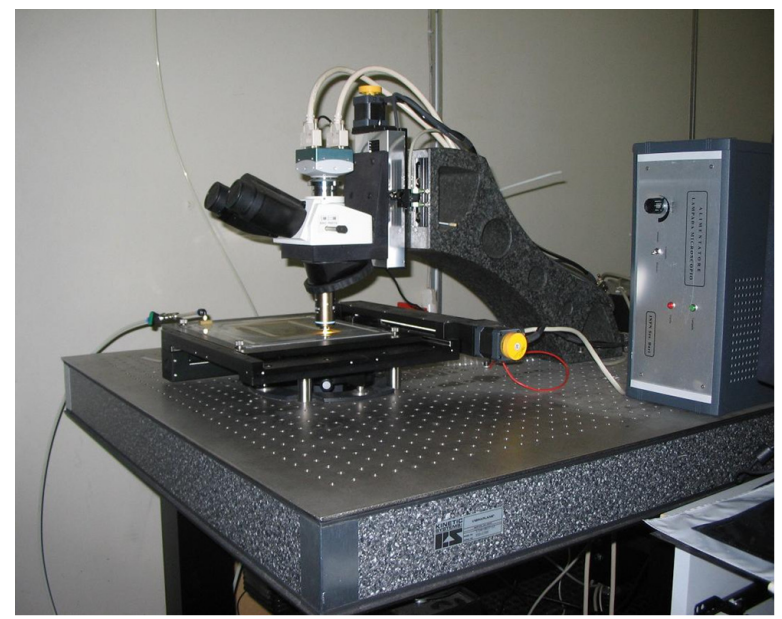

Figure 7. Prototype of the European scanning system for emulsion data acquisition.

monitoring was performed in order to control that the emulsion working temperature would be kept inside an acceptable range $\left(15^{\circ}-25^{\circ}\right)$. After the exposure, the detector was disassembled and the emulsions chemically developed. During the data taking, the emulsion films were held very tightly, in order to guarantee a pattern matching between consecutive films.

\subsection{Data analysis and preliminary results}

Emulsion readout is performed by fast automatic microscopes (Figure 7) [Arrabito et al. 2006] with a nominal scanning speed of $24 \mathrm{~cm}^{2} / \mathrm{h}$ recently upgraded to $40 \mathrm{~cm}^{2} / \mathrm{h}$ by keeping the current hardware configuration [Alexandrov 2013]. A sequence of 16 tomographic images is taken in each emulsion layer, with a step of $2.5 \mu \mathrm{m}$ in order to reconstruct the threedimensional tracks. All charged particle tracks, just after their production are recorded in each film up to the development phase, with no time information. The event time reconstruction is done offline by geometrical coincidences, that means at least two emulsion films are needed to get position pattern matching. In this way only tracks collected during the observation period match between adjacent films while the fake track coincidences due to combinatorial background are discarded. After the offline track reconstruction procedure widely detailed in Arrabito et al. [2007], it is possible to draw a bidimensional histogram with the reconstructed track density (Figure 8), TX and TY here are the directional tangents in respect to the telescope main axis. In this coordinate system the mountain contour visible by detector using the muons should coincide perfectly with the usual photo made from the same position. The color scale is the number of track found in each bin. The positive TY corresponds to tracks (muons) coming from the mountain direction, negative TY - muons comes from the back side (mainly from a free sky). The shadow of mountain is clearly visible for positive TY.

The free sky muon flux was computed on the basis of the HKKM04 model [Honda et al. 2004] with a cutoff for energies below $1 \mathrm{GeV}$ while in order to simulate the muon rates in the crater region we used the conventional cosmic ray flux attenuation values through the matter for each angle, and the digital elevation map (DEM) with $10 \mathrm{~m}$ resolution, kindly provided by the Italian Civil Defense. The DEM was also used to estimate the rock thickness shown in Figure 9d. On this angular plot the color scale for each bin presents the mean muon path (in meters) passed inside the rock before reaching the telescope.

The parameters used for the simulation are: a uniform rock density of $2.2 \mathrm{~g} / \mathrm{cm}^{3}$, an exposure time of 154 days and an effective detector surface of about $0.6 \mathrm{~m}^{2}$. The mountain profile is well visible and its shape is well in agreement with the simulation. It is also possible to observe, in the plot of Figure 9c, showing the difference between Monte Carlo and data, a 10\% excess of muon

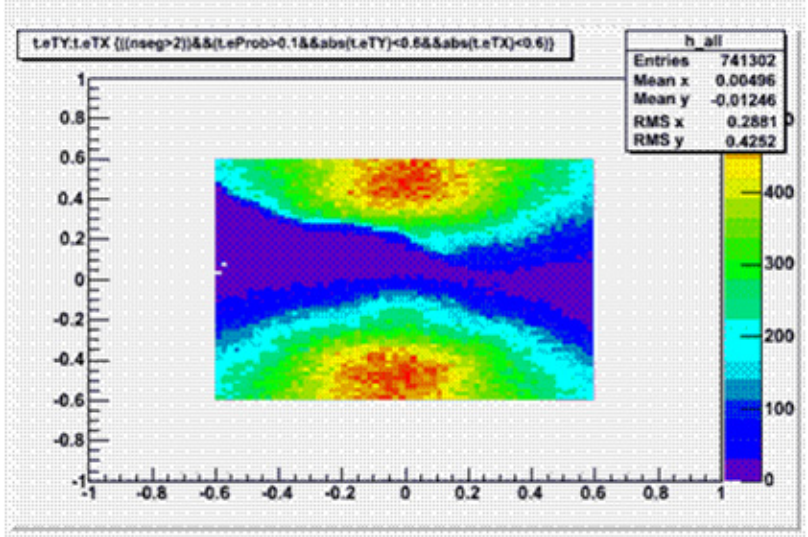

Figure 8. Bidimensional histogram of the counted muons in the angular space distribution obtained on a partial statistics. 

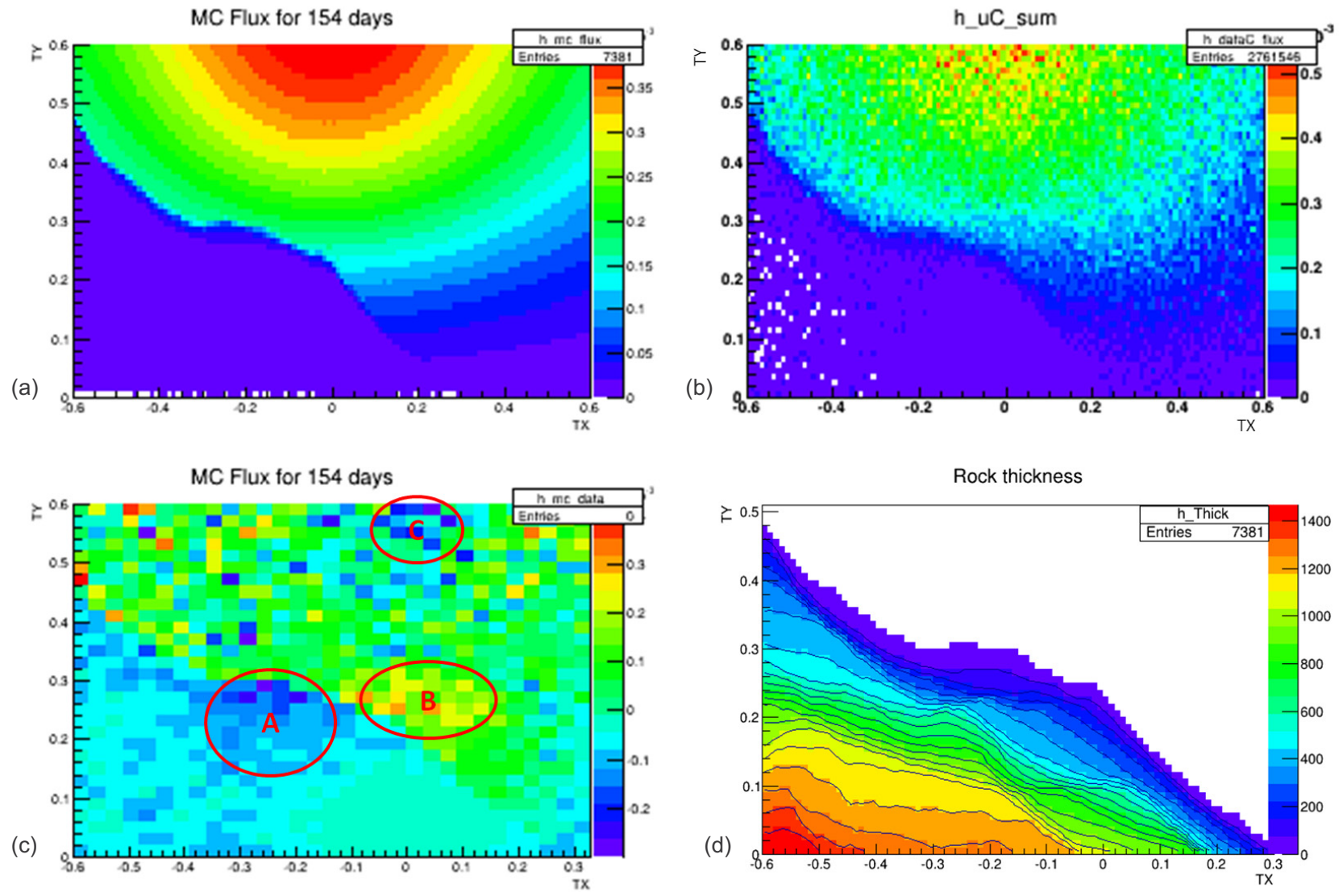

Figure 9. (a) Expected muon rates in 154 days on a detector surface of $0.6 \mathrm{~m}^{2}$; (b) observed muon rates; (c) difference normalized to the unit between Monte Carlo and data; (d) rock thickness.

counting rates in the rock region (red circle labelled A) which seems to reveal the presence of a crater. On the other side, the muon flux in the free sky shows some discrepancies respect to the expected one; this effect has to be better investigated performing deeper studies on the detection efficiency as a function of track momentum, choosing appropriate selection criteria in order to keep low momentum background tracks under control. Also a more precise Monte Carlo simulation taking into account the electromagnetic components of air showers and the cosmic muon scattering in the matter, should be provided. It is also necessary to consider the fact that the cosmic ray spectrum below $10 \mathrm{GeV}$ is affected by not negligible uncertainties due to several factors like geomagnetic latitude effects or solar modulation activity [Adriani et al. 2013]. At this stadium of the analysis it's not yet possible to infer finest effects; anyway the preliminary comparison between Monte Carlo and data seems to be promising.

\section{Conclusions and perspectives}

We described the muon radiography technique for imaging volcano structures, reporting the first preliminary results on the radiographic survey of Stromboli volcano with a nuclear emulsion telescope, obtained on the $60 \%$ of the collected statistics. A good match between the mountain shape and Monte Carlo simulation has been observed, that means a good understanding both of detector position and the topographic map usage. Simulated and observed data seem to be in a good agreement, but a more refined analysis is mandatory in order to better estimate the detection efficiency and background level for the final data interpretation. Actually the main background source as described in Nishiyama et al. [2014] is represented by low momentum tracks and one of the best tool to filter them is the usage of the Emulsion Cloud Chamber, OPERA-like that is made of interleaved layers of high- $Z$ plates and emulsion plates. Muon radiography of volcanoes with nuclear emulsions seems to be very encouraging and motivates further research to improve the capability of the detector, the scanning power and the ability in interpretating data also from a geological point of view aiming a closer cooperation with geologists. Italian laboratories are involved in other projects as the Mt. Teide exposure (2012) under analysis and the recent exposure in La Palma (2014) to investigate the shape of the fault i.e. the depth, the width, and the porosity of the crushing zone. Nuclear emulsions are really suitable for muon radiography applications for several aspects already mentioned. The main drawback in using such detectors is the huge amount of scanning power required, but such difficulty is on the way to be solved. The nominal scanning speed of automatic microscopes is about 24 
$\mathrm{cm}^{2} / \mathrm{h} /$ layer so the minimum time required for the full scanning of a Stromboli-like detector $\left(1 \mathrm{~m}^{2} \times 8\right.$ emulsion layers) was about 170 days using the scanning power equivalent to 2 microscopes working at $30 \%$ of the time. Thanks to the recent software and HW upgrades the scanning speed increased up to $80 \mathrm{~cm}^{2} / \mathrm{h} /$ layer. This value could be further raised up to more than 200 $\mathrm{cm}^{2} / \mathrm{h} /$ layer.

\section{References}

Acquafredda, R., et al. (2009). The OPERA experiment in the CERN to Gran Sasso neutrino beam, J. Instrum., 4, P04018.

Adriani, O., et al. (2013). Time dependence of the proton flux measured by PAMELA during the July 2006-December 2009 solar minimum, Astrophys. J., 765, 91.

Agafonova, N., et al. (2010). Observation of a first $\nu_{\tau}$ candidate in the OPERA experiment in the CNGS beam, Phys. Lett. B, 691, 138-145.

Agafonova, N., et al. (2013). New results on $v_{\mu} \rightarrow v_{\tau}$ appearance with the OPERA experiment in the CNGS beam, J. High Energy Phys., 1311036.

Agafonova, N., et al. (2014). Evidence for $v_{\mu} \rightarrow v_{\tau}$ appearance in the CNGS neutrino beam with the OPERA experiment, Phys. Rev. D., 89, 051102.

Alexandrov, A. (2013). A novel approach for fast scanning of nuclear emulsions with continuous motion of the microscope stage, Conference Proceedings, Nucl. Instrum. Meth A, 718, 184-185.

Arrabito, L., et al. (2006). Hardware performance of a scanning system for high speed analysis of nuclear emulsions, Nucl. Instrum. Meth. A, 568, 578-587.

Arrabito, L., et al. (2007). Track reconstruction in the emulsion-lead target of the OPERA experiment using the ESS microscope, 2, P05004.

Auger, E., L. D'Auria, M. Martini, B. Chouet and P. Dawson (2006). Realtime monitoring and massive inversion of source parameters of very long period seismic signals: An application to Stromboli Volcano, Geophys. Res. Lett., 33, L04301; doi:10.1029/2005 GL024703.

Chouet, B., P. Dawson, T. Ohminato, M. Martini, G. Saccorotti, F. Giudicepietro, G. De Luca, G. Milana and R. Scarpa (2007). Source mechanisms of explosions at Stromboli Volcano determined from moment-tensor inversions of very-longperiod data, J. Geophys. Res., 108 (B1), 2019; doi:10.1029/2002JB 001919.

Gaisser, T. (1990). Cosmic Rays and Particle Physics, Cambridge University Press, New York.

Honda, M., T. Kajita, K. Kasahara and S. Midorikawa (2004). New calculation of the atmospheric neutrino flux in a three-dimensional scheme, Phys. Rev. D, $70,043008$.

Macedonio, G. (2009). Motivations for muon radiography of active volcanoes, Earth Planets Space, 61, 1-5.

Nakamura, T., et al. (2006). The OPERA film: New nuclear emulsion for large-scale, high-precision experiments, Nucl. Instrum. Meth. A, 556, 80-86.

Nishiyama, R., S. Miyamoto and N. Naganawa (2014). Experimental study of source of background noise in muon radiography using emulsion film detectors, Geosci. Instrum. Meth., 3, 29-39.

Tanaka, H.K.M., T. Nakano, S. Takahashi, J. Yoshida and K. Niwa (2007a). Development of an emulsion imaging system for cosmic-ray muon radiography to explore the internal structure of a volcano, Mt. Asama, Nucl. Instrum. Meth. A, 575, 489-497.

Tanaka, H.K.M., T. Nakano, S. Takahashi, J. Yoshida, H. Ohshima, T. Maekawa, H. Watanabe and K. Niwa (2007b). Imaging the conduit size of the dome with cosmic ray muons: The structure beneath Showa Shinzan Lava Dome, Japan, Geophys. Res. Lett., 34, L22311.

\footnotetext{
${ }^{\star}$ Corresponding author: Valeri Tioukov, Istituto Nazionale di Fisica Nucleare (INFN), Sezione di Napoli, Naples, Italy; email: valeri@na.infn.it.

C 2017 by the Istituto Nazionale di Geofisica e Vulcanologia. All rights reserved.
} 